\title{
Emergency Telepsychiatry and the Acute Care Continuum: Creating Value Through Improved Patient Accessibility and Follow-up
}

Prentice A. Tom

Editor's note: Dr. Tom is Chief Innovation Officer and Executive Vice President of Medical Affairs at CEP America. This is the first in a series of articles that will appear in Telehealth and Medicine Today in which he will share his view of development and implementation of healthcare service solutions.

I few years ago, I coined the term, "Medicine Without Walls"1 to describe our future healthcare delivery system-an environment where patient and healthcare practitioner are unencumbered by physical location or limitations in access points due to human resource restrictions, where medical information is transferred not only between patient and clinician but also between any number of care practitioners and healthcare institutions - a world where patients have open and ready access to medical care when and where needed.

The acute care continuum is the space where telemedicine can most impact healthcare delivery. The term, "acute care continuum," refers to all care, including the penumbra of care surrounding higher severity acute medical events, which requires urgent, emergent, hospital and/or surgicenter services.

CEP America has found significant opportunity to use telemedicine to improve access, improve care, and reduce cost and resource utilization in these environments.

To this end, a number of telehealth solutions have been created that augment our ability to provide timely, necessary, and improved care: emergency telepsychiatry, postdischarge/skilled nursing facilities (SNF) and urgent care. In future articles, updates on our SNF and urgent care telemedicine programs will be presented. This article focuses on emergency telepsychiatry. 


\section{Emergency Telepsychiatry}

Emergency care, psychiatric emergency services and telepsychiatry provide an excellent example of how telehealth technology can be leveraged to create a population health solution that integrates care and improves outcomes. Despite the fact that one in eight emergency department (ED) patients has a psychiatric related condition ${ }^{2}$, access to necessary emergency psychiatric care is recognized as one of our nation's most difficult care issues. ${ }^{3}$

Over the last two decades, advances in patient flow processes have resulted in consistent reductions in emergency department wait times. Still, because of the severe nationwide shortage of psychiatric beds, time to definitive care for this unique group of patients can be delayed for an unconscionable two or even three days (vs. 160/279 minutes [discharged/admitted] for the average ED patient), ${ }^{4}$

\section{Case Presentation}

Through the creation of an integrated population health approach for ED patients, which incorporates telepsychiatry as the key mechanism to achieving rapid early assessment, wait times for emergency psychiatric patients were reduced by over $50 \%$. Our first telepsychiatry program was implemented in March 2016 and rapidly expanded to four hospitals, with start dates in June and July 2016. In 2016, 1479 patients were treated through our various telepsychiatry programs at four community hospitals. We now average approximately 280 patients per month through these existing programs and anticipate expanding this program to other client hospitals and health systems in the coming months.

Prior to implementing our telepsychiatry program, a study of emergency psychiatry patients at these four hospitals showed an average time to discharge of 439 minutes, and an average time to admission of 1,267 minutes. After implementation, the average time to discharge dropped to 388 minutes (a 12\% improvement), and the average time to admission dropped to 531 minutes (a $58 \%$ improvement). ${ }^{5}$ 
An ED physician's summary of a case that illustrates the benefit of this emergency telepsychiatry program is provided below.

I was working in the Emergency Department and received a call from one of our primary care physicians (PCPs). The PCP had a young male with history of schizophrenia in his office that he felt needed to be further evaluated in the ED. The ED was completely packed, and we were already holding multiple admitted patients. The PCP called the police and had the patient placed in custody and transported to the ED on a hold.

The patient was evaluated by myself. He reported a previous diagnosis of "schizophrenia", but was actually taking medications for depression and was not taking any antipsychotics. He did not seem internally occupied, did not have a flat affect, and was able to interact with police, the nurse, and myself in a normal manner. I did not think he was psychotic and doubted the diagnosis of acute debilitating schizophrenia. Unfortunately, it was late in the evening, and if not for the availability of telepsychiatry, he would have to remain on a hold and admitted to our holding area until I could obtain definitive psychiatric confirmation. Given we have no psychiatry call available on the weekends, if not for our telepsychiatry program, he would not have been seen until the coming Monday.

Instead, I placed a consult to our telepsychiatrist, explained the case, and moved on to the next patient. That physician spent the next hour evaluating the patient. He provided a consult note describing in detail how the patient's presentation was inconsistent with a diagnosis of schizophrenia or any emergent debilitating psychiatric condition, and as a result, I was able to send the patient home.

If not for our telepsychiatry program, this patient would have languished in our ED for possibly three to four days, but instead was discharged in under two hours. 


\section{Discussion: Application of Telepsychiatry Benefits for Stakeholders in Healthcare}

As a result (in part) of this experience, improved care resulting from our telepsychiatry program, are being instituted and expanded at a number of our practice locations.

To successfully deploy a telehealth offering, focus should be on efforts around what matters most-the best quality care provider, the operations, the patient experience, the care coordination, and the nuances of your local healthcare ecosystem that will make or break your deployment. Patients will thank you for making "Medicine without Walls" a reality for them and their families.

Our nation's EDs are often the first filter for ensuring that patients with emergent psychiatric conditions do not pose an immediate threat to themselves or others. Accordingly, emergency physicians, law enforcement, and others able to designate patients as "holdable" pending definitive psychiatric evaluation typically have a low threshold and high sensitivity for identifying such patients. As a result, there is evidence that suggests $70 \%$ of emergency patients admitted or transferred to a psychiatric hospital for definitive care can be cleared and safely discharged by a psychiatrist without needing longer-term in-patient treatment. ${ }^{6}$ Emergency psychiatric evaluation is particularly amenable to telemedicine technology, which can often be deployed early in the patient's care. The impact can be enormous for patients, practitioners, healthcare systems, payers, and psychiatric hospitals.

For patients, telepsychiatry results in reduced stay in a stressful noisy environment that can contribute to patient anxiety. For the care practitioner, telepsychiatry can help ameliorate risk by ensuring more rapid referral of patients that truly need definitive emergent care.

For healthcare systems, it frees up ED beds, allowing for increased volume resulting in capture of opportunity cost for the hospital as well as more timely care for other patients awaiting an ED bed. For payers, emergency telepsychiatry reduces costs by more 
appropriately allocating necessary resources to only those patients that require transfer and admission to psychiatric hospitals.

And, for receiving psychiatric hospitals, telepsychiatry reduces the patient load burden by identifying which referred patients are truly emergent, thus allowing the specialty facility to both avoid admission denials and more appropriately prepare for and focus on those patients truly needing in-patient psychiatric care.

\section{Conclusions}

Various management components are necessary to successful implement a telemedicine program, with clinician management including integration with the health system the most difficult. It is critical to ensure that the telemedicine company is experienced in partnering with clinician groups and an understanding of unique local health system needs, with the ability to adapt their product to accommodate the demands of integrating telemedicine into a larger often non-integrated health environment. We partnered with a highly respected telemedicine company able to address the varying needs of emergency psychiatry, chronic disease management, and urgent care clinicians working in vastly different environments and systems to provide the necessary technical expertise.

As with in-person care, the crucial components for a successful telehealth offering are managing clinicians and integrating various non-aligned care provider groups, payers, and health systems. This is a daunting task and complexity. It should not be underestimated by stakeholders. For example, key to CEP America's successful rollouts of various telemedicine programs are program-specific intensive clinician training, with educational content on clinician telecommunications presence and communication techniques, telemedicine adapted clinical pathways, and the limits of care that can be safely provided through a telemedicine venue.

Advances in health-related technologies (more than changes in healthcare financing) including interoperability, the many benefits of big data, advances in point-of-care 
testing, and especially telemedicine are converging to make this once utopian dream our reality. As Chief Innovation Officer of one of our nation's largest acute care continuum physician services organization, I find it incredibly rewarding to help guide CEP America's contributions, through our telemedicine programs, towards this future paradigm of "Medicine Without Walls".

Although early in implementation, our acute care continuum telemedicine programs are showing significant success (as evidenced by this report) in decreasing patient access barriers to care, improving resource allocation efficiencies, improving timeliness of care, improving access to high demand medical services, improving patient follow-up and outcomes, improving patient experience with their health system encounter, improving care provider satisfaction and work experience, and reducing healthcare expenditures.

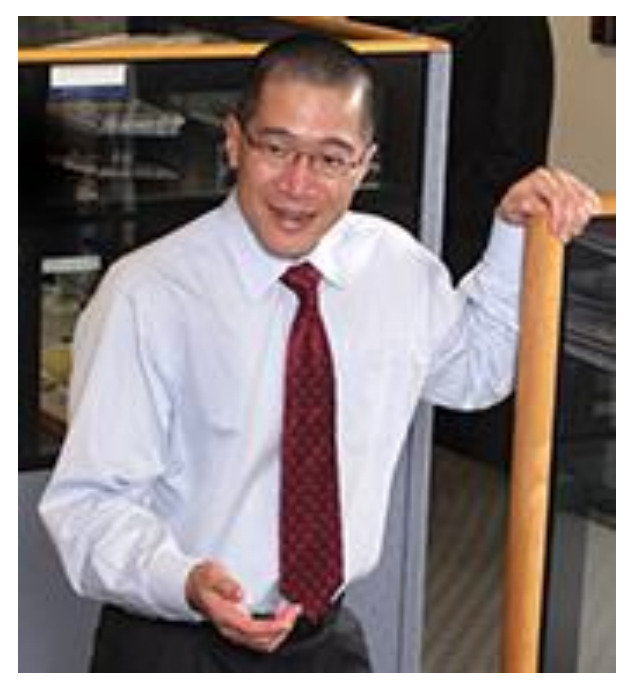

Prentice A. Tom, MD, FACEP, is Chief Innovation Officer and Executive Vice President of Medical Affairs at CEP America. In this role, he oversees the development and implementation of healthcare service solutions.

\section{References}

1. Author presentation, Medicine without Walls, CEP America Spring Symposium, San Francisco, Los Angeles and Chicago, April 2015, and published in Urgent Matters, July 
2015: https://smhs.gwu.edu/urgentmatters/news/medicine-without-walls-populationhealth-and-acute-care-continuum.

2 US Department of Health and Human Services. Mental disorders and/or substance abuse related to one of every eight emergency department cases: AHRQ news and numbers. July 2010. Agency for Healthcare Research and Quality, Rockville, MD. http://archive.ahrq.gov/news/newsroom/news-and-numbers/070810.html 3. Bender D, Pande N, Lugwig M. A literature review: Psychiatric boarding. ASPE. 2008. URL: https://aspe.hhs.gov/basic-report/literature-review-psychiatricboarding\#conclude. Accessed 4/4/17.

4. CMS Hospital Compare Data, 04/01/15, https://data.medicare.gov/HospitalCompare/Timely-and-Effective-Care-National/isrn-hqyy.

5 Brokmann JC, Rossaint R, Bergrath S, et al. [Potential and effectiveness of a telemedical rescue assistance system. Prospective observational study on implementation in emergency medicine]. Anesthetist. 2015 Jun;64(6):438-45.

6. Zeller, S.; Calma, N.; Stone, A. Effects of a Regional Dedicated Psychiatric Emergency Service on Boarding and Hospitalization of Psychiatric Patients in Area Emergency Departments. Western Journal of Emergency Medicine 2014; 15.

Dept. Interviews \& Opinions

Tags: CEP America, emergency care, Prentice A. Tom, psychiatric emergency services, telepsychiatry 